IJMMS 27:12 (2001) 749-757

PII. S0161171201006627

http://ijmms.hindawi.com

(C) Hindawi Publishing Corp.

\title{
ON $Q$-ALGEBRAS
}

\section{JOSEPH NEGGERS, SUN SHIN AHN, and HEE SIK KIM}

\author{
(Received 29 January 2001)
}

\begin{abstract}
We introduce a new notion, called a $Q$-algebra, which is a generalization of the idea of $B C H / B C I / B C K$-algebras and we generalize some theorems discussed in $B C I$ algebras. Moreover, we introduce the notion of "quadratic" $Q$-algebra, and show that every quadratic $Q$-algebra $(X ; *, e), e \in X$, has a product of the form $x * y=x-y+e$, where $x, y \in X$ when $X$ is a field with $|X| \geq 3$.
\end{abstract}

2000 Mathematics Subject Classification. 06F35, 03G25.

1. Introduction. Imai and Iséki introduced two classes of abstract algebras: $B C K$ algebras and $B C I$-algebras (see $[4,5]$ ). It is known that the class of $B C K$-algebras is a proper subclass of the class of $B C I$-algebras. In [2, 3] Hu and Li introduced a wide class of abstract algebras: $B C H$-algebras. They have shown that the class of $B C I$-algebras is a proper subclass of the class of $B C H$-algebras. Neggers and Kim (see [8]) introduced the notion of $d$-algebras, that is, (I) $x * x=e$; (IX) $e * x=e$; (VI) $x * y=e$ and $y * x=e$ imply $x=y$, which is another useful generalization of $B C K$-algebras, after which they investigated several relations between $d$-algebras and $B C K$-algebras, as well as other relations between $d$-algebras and oriented digraphs. At the same time, Jun, Roh, and Kim [6] introduced a new notion, called a $B H$-algebra, that is, (I) $x * x=e$; (II) $x * e=x$; (VI) $x * y=e$ and $y * x=e$ imply $x=y$, which is a generalization of $B C H / B C I / B C K$-algebras, and they showed that there is a maximal ideal in bounded $B H$-algebras. We introduce a new notion, called a $Q$-algebra, which is a generalization of $B C H / B C I / B C K$-algebras and generalize some theorems from the theory of $B C I$ algebras. Moreover, we introduce the notion of "quadratic" $Q$-algebra, and obtain the result that every quadratic $Q$-algebra $(X ; *, e), e \in X$, is of the form $x * y=x-y+e$, where $x, y \in X$ and $X$ is a field with $|X| \geq 3$, that is, the product is linear in a special way.

2. $Q$-algebras. A $Q$-algebra is a nonempty set $X$ with a constant 0 and a binary operation " $*$ " satisfying axioms:

(I) $x * x=0$,

(II) $x * 0=x$,

(III) $(x * y) * z=(x * z) * y$ for all $x, y, z \in X$.

For brevity we also call $X$ a $Q$-algebra. In $X$ we can define a binary relation $\leq$ by $x \leq y$ if and only if $x * y=0$. Recently, Ahn and Kim [1] introduced the notion of $Q S$-algebras. A $Q$-algebra $X$ is said to be a $Q S$-algebra if it satisfies the additional relation:

(IV) $(x * y) *(x * z)=z * y$, for any $x, y, z \in X$ 
EXAMPLE 2.1. Let $\mathbb{Z}$ be the set of all integers and let $n \mathbb{Z}:=\{n z \mid z \in \mathbb{Z}\}$ where $n \in \mathbb{Z}$. Then $(\mathbb{Z} ;-, 0)$ and $(n \mathbb{Z} ;-, 0)$ are $Q$-algebras, where "-" is the usual subtraction of integers.

EXAMPLE 2.2. Let $X:=\{0,1,2,3\}$ be a set with the following table:

\begin{tabular}{l|llll}
\hline$*$ & 0 & 1 & 2 & 3 \\
\hline 0 & 0 & 0 & 0 & 0 \\
1 & 1 & 0 & 0 & 0 \\
2 & 2 & 0 & 0 & 0 \\
3 & 3 & 3 & 3 & 0 \\
\hline
\end{tabular}

Then $(X ; *, 0)$ is a $Q$-algebra, which is not a $B C H / B C I / B C K$-algebra.

Neggers and Kim [7] introduced the related notion of $B$-algebra, that is, algebras $(X ; *, 0)$ which satisfy (I) $x * x=0$; (II) $x * 0=x$; (V) $(x * y) * z=x *(z *(0 * y))$, for any $x, y, z \in X$. It is easy to see that $B$-algebras and $Q$-algebras are different notions. For example, Example 2.2 is a $Q$-algebra, but not a $B$-algebra, since $(3 * 2) * 1=0 \neq$ $3=3 *(1 *(0 * 2))$. Consider the following example. Let $X:=\{0,1,2,3,4,5\}$ be a set with the following table:

\begin{tabular}{l|llllll}
\hline$*$ & 0 & 1 & 2 & 3 & 4 & 5 \\
\hline 0 & 0 & 2 & 1 & 3 & 4 & 5 \\
1 & 1 & 0 & 2 & 4 & 5 & 3 \\
2 & 2 & 1 & 0 & 5 & 3 & 4 \\
3 & 3 & 4 & 5 & 0 & 2 & 1 \\
4 & 4 & 5 & 3 & 1 & 0 & 2 \\
5 & 5 & 3 & 4 & 2 & 1 & 0 \\
\hline
\end{tabular}

Then $(X ; *, 0)$ is a $B$-algebra (see [7]), but not a $Q$-algebra, since $(5 * 3) * 4=3 \neq 4=$ $(5 * 4) * 3$.

Proposition 2.3. If $(X ; *, 0)$ is a $Q$-algebra, then

(VII) $(x *(x * y)) * y=0$, for any $x, y \in X$.

Proof. By (I) and (III), $(x *(x * y)) * y=(x * y) *(x * y)=0$.

We now investigate some relations between $Q$-algebras and $B C H$-algebras (also $B C K / B C I$-algebras). The following theorems are easily proven, and we omit their proofs.

THEOREM 2.4. Every BCH-algebra $X$ is a $Q$-algebra. Every $Q$-algebra $X$ satisfying condition (VI) is a BCH-algebra.

THEOREM 2.5. Every Q-algebra satisfying condition (IV) and (VI) is a BCI-algebra. 
THEOREM 2.6. Every $Q$-algebra $X$ satisfying conditions (V), (VI), and (VIII) $(x * y) * x=0$ for any $x, y \in X$, is a BCK-algebra.

THEOREM 2.7. Every $Q$-algebra $X$ satisfying $x *(x * y)=x * y$ for all $x, y, z \in X$, is a trivial algebra.

Proof. Putting $x=y$ in the equation $x *(x * y)=x * y$, we obtain $x * 0=0$. By (II) $x=0$. Hence $X$ is a trivial algebra.

The following example shows that a $Q$-algebra may not satisfy the associative law.

EXAMPLE 2.8. (a) Let $X:=\{0,1,2\}$ with the table as follows:

\begin{tabular}{l|lll}
\hline$*$ & 0 & 1 & 2 \\
\hline 0 & 0 & 2 & 1 \\
1 & 1 & 0 & 2 \\
2 & 2 & 1 & 0 \\
\hline
\end{tabular}

Then $X$ is a $Q$-algebra, but associativity does not hold, since $(0 * 1) * 2=0 \neq 1=$ $0 *(1 * 2)$.

(b) Let $\mathbb{Z}$ and $\mathbb{R}$ be the set of all integers and real numbers, respectively. Then $(\mathbb{Z} ;-, 0)$ and $(\mathbb{R} ; \div, 1)$ are nonassociative $Q$-algebras where "-" is the usual subtraction and " $\div$ " is the usual division.

THEOREM 2.9. Every $Q$-algebra $(X ; *, 0)$ satisfying the associative law is a group under the operation “*”.

Proof. Putting $x=y=z$ in the associative law $(x * y) * z=x *(y * z)$ and using (I) and (II), we obtain $0 * x=x * 0=x$. This means that 0 is the zero element of $X$. By (I), every element $x$ of $X$ has as its inverse the element $x$ itself. Therefore $(X ; *)$ is a group.

3. The $G$-part of $Q$-algebras. In this section, we investigate the properties of the $G$-part in $Q$-algebras.

LEMmA 3.1. If $(X ; *, 0)$ is $a Q$-algebra and $a * b=a * c, a, b, c \in X$, then $0 * b=0 * c$.

Proof. By (I) and (II) $(a * b) * a=(a * a) * b=0 * b$ and $(a * c) * a=(a * a) * c=$ $0 * c$. Since $a * b=a * c, 0 * b=0 * c$.

DeFinITION 3.2. Let $(X ; *, 0)$ be a $Q$-algebra. For any nonempty subset $S$ of $X$, we define

$$
G(S):=\{x \in S \mid 0 * x=x\} .
$$

In particular, if $S=X$ then we say that $G(X)$ is the $G$-part of $X$.

COROLLARY 3.3. A left cancellation law holds in $G(X)$.

Proof. Let $a, b, c \in G(X)$ with $a * b=a * c$. By Lemma 3.1, $0 * b=0 * c$. Since $b, c \in G(X)$, we obtain $b=c$. 
Proposition 3.4. Let $(X ; *, 0)$ be a $Q$-algebra. Then $x \in G(X)$ if and only if $0 * x \in$ $G(X)$.

Proof. If $x \in G(X)$, then $0 * x=x$ and $0 *(0 * x)=0 * x$. Hence $0 * x \in G(X)$.

Conversely, if $0 * x \in G(x)$, then $0 *(0 * x)=0 * x$. By applying Corollary 3.3, we obtain $0 * x=x$. Therefore $x \in G(X)$.

For any $Q$-algebra $(X ; *, 0)$, the set

$$
B(X):=\{x \in X \mid 0 * x=0\}
$$

is called the $p$-radical of $X$. If $B(X)=\{0\}$, then we say that $X$ is a $p$-semisimple $Q$-algebra. The following property is obvious.

(IX) $G(X) \cap B(X)=\{0\}$.

Proposition 3.5. If $(X ; *, 0)$ is a $Q$-algebra and $x, y \in X$, then

$$
y \in B(X) \Longleftrightarrow(x * y) * x=0 .
$$

Proof. By (I) and (III) $(x * y) * x=(x * x) * y=0 * y=0$ if and only if $y \in B(X$ ए

DEFINITION 3.6. Let $(X ; *, 0)$ be a $Q$-algebra and $I(\neq \varnothing) \subseteq X$. The set $I$ is called an ideal of $X$ if for any $x, y, z \in X$,

(1) $0 \in I$,

(2) $x * y \in I$ and $y \in I$ imply $x \in I$.

Obviously, $\{0\}$ and $X$ are ideals of $X$. We call $\{0\}$ and $X$ the zero ideal and the trivial ideal of $X$, respectively. An ideal $I$ is said to be proper if $I \neq X$.

In Example 2.2 the set $I:=\{0,1,2\}$ is an ideal of $X$.

Proposition 3.7. Let $(X ; *, 0)$ be a $Q$-algebra. Then $B(X)$ is an ideal of $X$.

Proof. Since $(0 * 0) * 0=0$, by Proposition 3.5, $0 \in B(X)$. Let $x * y \in B(X)$ and $y \in B(X)$. Then by Proposition 3.5, $((x * y) * x) *(x * y)=0$. By (III), $((x * y) *(x *$ $y)) * x=0 * x=0$. Hence $x \in B(X)$. Therefore $B(X)$ is an ideal of $X$.

Proposition 3.8. If $S$ is a subalgebra of a $Q$-algebra $(X ; *, 0)$, then $G(X) \cap S=G(S)$.

Proof. It is obvious that $G(X) \cap S \subseteq G(S)$. If $x \in G(S)$, then $0 * x=x$ and $x \in S \subseteq$ $X$. Then $x \in G(X)$ and so $x \in G(X) \cap S$, which proves the proposition.

THEOREM 3.9. Let $(X ; *, 0)$ be a $Q$-algebra. If $G(X)=X$, then $X$ is $p$-semisimple.

Proof. Assume that $G(X)=X$. By $(X),\{0\}=G(X) \cap B(X)=X \cap B(X)=B(X)$. Hence $X$ is $p$-semisimple.

THEOREM 3.10. If $(X ; *, 0)$ is a $Q$-algebra of order 3 , then $|G(X)| \neq 3$, that is, $G(X) \neq X$.

Proof. For the sake of convenience, let $X=\{0, a, b\}$ be a $Q$-algebra. Assume that $|G(X)|=3$, that is, $G(X)=X$. Then $0 * 0=0,0 * a=a$, and $0 * b=b$. From $x * x=0$ and $x * 0=x$, it follows that $a * a=0, b * b=0, a * 0=a$, and $b * 0=b$. Now let $a * b=0$. Then $0, a$, and $b$ are candidates of the computation. If $b * a=0$, then 
$a * b=0=b * a$ and so $(a * b) * a=(b * a) * a$. By (III), $(a * a) * b=(b * a) * a$. Hence $0 * b=0 * a$. By the cancellation law in $G(X), b=a$, a contradiction. If $b * a=a$, then $a=b * a=(0 * b) * a=(0 * a) * b=a * b=0$, a contradiction. For the case $b * a=b$, we have $b=b * a=(0 * b) * a=(0 * a) * b=a * b=0$, which is also a contradiction. Next, if $a * b=a$, then $(a *(a * b)) * b=(a * a) * b=0 * b=b \neq 0$. This leads to the conclusion that Proposition 2.3 does not hold, a contradiction. Finally, let $a * b=b$. If $b * a=0$, then $b=a * b=(0 * a) * b=(0 * b) * a=b * a=0$, a contradiction. If $b * a=a, b=a * b=(0 * a) * b=(0 * b) * a=b * a=0$, a contradiction. For the case $b * a=b$, we have $a=0 * a=(b * b) * a=(b * a) * b=b * b=0$, which is again a contradiction. This completes the proof.

Proposition 3.11. If $(X ; *, 0)$ is a $Q$-algebra of order 2 , then in every case the $G$-part $G(X)$ of $X$ is an ideal of $X$.

Proof. Let $|X|=2$. Then either $G(X)=\{0\}$ or $G(X)=X$. In either case, $G(X)$ is an ideal of $X$.

THEOREM 3.12. Let $(X ; *, 0)$ be a $Q$-algebra of order 3 . Then $G(X)$ is an ideal of $X$ if and only if $|G(X)|=1$.

Proof. Let $X:=\{0, a, b\}$ be a $Q$-algebra. If $|G(X)|=1$, then $G(X)=\{0\}$ is the trivial ideal of $X$.

Conversely, assume that $G(X)$ is an ideal of $X$. By Theorem 3.10, we know that either $|G(X)|=1$ or $|G(X)|=2$. Suppose that $|G(X)|=2$. Then either $G(X)=\{0, a\}$ or $G(X)=\{0, b\}$. If $G(X)=\{0, a\}$, then $b * a \notin G(X)$ because $G(X)$ is an ideal of $X$. Hence $b * a=b$. Then $a=0 * a=(b * b) * a=(b * a) * b=b * b=0$, which is a contradiction. Similarly, $G(X)=\{0, b\}$ leads to a contradiction. Therefore $|G(X)| \neq 2$ and so $|G(X)|=1$.

DEFINITION 3.13. An ideal $I$ of a $Q$-algebra $(X ; *, 0)$ is said to be implicative if $(x * y) * z \in I$ and $y * z \in I$, then $x * z \in I$, for any $x, y, z \in X$.

THEOREM 3.14. Let $(X ; *, 0)$ be a $Q$-algebra and let I be an implicative ideal of $X$. Then I contains the $G$-part $G(X)$ of $X$.

Proof. If $x \in G(X)$, then $(0 * x) * x=x * x=0 \in I$ and $x * x=0 \in I$. Since $I$ is implicative, it follows that $x=0 * x \in I$. Hence $G(X) \subseteq I$.

DeFinITION 3.15. Let $X$ and $Y$ be $Q$-algebras. A mapping $f: X \rightarrow Y$ is called a homomorphism if

$$
f(x * y)=f(x) * f(y), \quad \forall x, y \in X .
$$

A homomorphism $f$ is called a monomorphism (resp., epimorphism) if it is injective (resp., surjective). A bijective homomorphism is called an isomorphism. Two $Q$ algebras $X$ and $Y$ are said to be isomorphic, written by $X \cong Y$, if there exists an isomorphism $f: X \rightarrow Y$. For any homomorphism $f: X \rightarrow Y$, the set $\{x \in X \mid f(x)=0\}$ is called the kernel of $f$, denoted by $\operatorname{Ker}(f)$ and the set $\{f(x) \mid x \in X\}$ is called the image of $f$, denoted by $\operatorname{Im}(f)$. We denote by $\operatorname{Hom}(X, Y)$ the set of all homomorphisms of $Q$-algebras from $X$ to $Y$. 
Proposition 3.16. Suppose that $f: X \rightarrow X^{\prime}$ is a homomorphism of Q-algebras. Then

(1) $f(0)=0^{\prime}$,

(2) $f$ is isotone, that is, if $x * y=0, x, y \in X$, then $f(x) * f(y)=0^{\prime}$.

Proof. Since $f(0)=f(0 * 0)=f(0) * f(0)=0^{\prime}$, (1) holds. If $x, y \in X$ and $x \leq y$, that is, $x * y=0$, then by $(1), f(x) * f(y)=f(x * y)=f(0)=0^{\prime}$. Hence $f(x) \leq f(y)$, proving (2).

THEOREM 3.17. Let $(X ; *, 0)$ and $\left(X ; *^{\prime}, 0^{\prime}\right)$ be $Q$-algebras and let $B$ be an ideal of $Y$. Then for any $f \in \operatorname{Hom}(X, Y), f^{-1}(B)$ is an ideal of $X$.

Proof. By Proposition 3.16(1), $0 \in f^{-1}(B)$. Assume that $x * y \in f^{-1}(B)$ and $y \in$ $f^{-1}(B)$. Then $f(x) * f(y)=f(x * y) \in B$. It follows from the fact that $B$ is an ideal of $Y$ that $f(x) \in B$, that is, $x \in f^{-1}(B)$. This means that $f^{-1}(B)$ is an ideal of $X$. The proof is complete.

Since $\left\{0^{\prime}\right\}$ is an ideal of $X^{\prime}, \operatorname{Ker}(f)=f^{-1}\left(\left\{0^{\prime}\right\}\right)$ for any $f \in \operatorname{Hom}(X, Y)$. Hence we obtain the following corollary.

COROLlarY 3.18. The kernel $\operatorname{Ker}(f)$ is an ideal of $X$.

4. The quadratic $Q$-algebras. Let $X$ be a field with $|X| \geq 3$. An algebra $(X ; *)$ is said to be quadratic if $x * y$ is defined by $x * y:=a_{1} x^{2}+a_{2} x y+a_{3} y^{2}+a_{4} x+a_{5} y+a_{6}$, where $a_{1}, \ldots, a_{6} \in X$, for any $x, y \in X$. A quadratic algebra $(X ; *)$ is said to be quadratic $Q$-algebra (resp., $Q S$-algebra) if it satisfies conditions (I), (II), and (III) (resp., (IV)).

THEOREM 4.1. Let $X$ be a field with $|X| \geq 3$. Then every quadratic $Q$-algebra $(X ; *, e)$, $e \in X$, has the form $x * y=x-y+e$ where $x, y \in X$.

Proof. Define

$$
x * y:=A x^{2}+B x y+C y^{2}+D x+E y+F
$$

Consider (I).

$$
e=x * x=(A+B+C) x^{2}+(D+E) x+F .
$$

Let $x:=0$ in (4.2). Then we obtain $F=e$. Hence (4.1) turns out to be

$$
x * y=A x^{2}+B x y+C y^{2}+D x+E y+e .
$$

If $y:=x$ in (4.3), then

$$
e=x * x=(A+B+C) x^{2}+(D+E) x+e,
$$

for any $x \in X$, and hence we obtain $A+B+C=0=D+E$, that is, $E=-D$ and $B=$ $-A-C$. Hence (4.3) turns out to be

$$
x * y=(x-y)(A x-C y+D)+e .
$$

Let $y:=e$ in (4.5). Then by (II) we have

$$
x=x * e=(x-e)(A x-C e+D)+e,
$$


that is, $(A x-C e+D-1)(x-e)=0$. Since $X$ is a field, either $x-e=0$ or $A x-C e+$ $D-1=0$. Since $|X| \geq 3$, we have $A x-C e+D-1=0$, for any $x \neq e$ in $X$. This means that $A=0,1-D+C e=0$. Thus (4.5) turns out to be

$$
x * y=(x-y)+C(x-y)(e-y)+e .
$$

To satisfy condition (III) we consider $(x * y) * z$ and $(x * z) * y$.

$$
\begin{aligned}
(x * y) * z= & (x * y-z)+C(x * y-z)(e-z)+e \\
= & (x-y-z)+C(x-y)(e-z)+2 e \\
& +C[(x-y)+C(x-y)(e-y)+(e-z)](e-z) \\
= & (x-y-z)+C(x-y)(2 e-y-z)+2 e \\
& +C^{2}(x-y)(e-y)(e-z)+C(e-z)^{2} .
\end{aligned}
$$

Interchange $y$ with $z$ in (4.8). Then

$$
\begin{aligned}
(x * z) * y= & (x-z-y)+C(x-z)(2 e-z-y)+2 e \\
& +C^{2}(x-z)(e-z)(e-y)+C(e-y)^{2} .
\end{aligned}
$$

By (4.8) and (4.9) we obtain

$$
0=(x * y) * z-(x * z) * y=C^{2}(e-y)(e-z)(z-y) .
$$

Since $X$ is a field with $|X| \geq 3$, we obtain $C=0$. This means that every quadratic $Q$-algebra $(X ; *, e)$, has the form $x * y=x-y+e$ where $x, y \in X$, completing the proof.

EXAMPLE 4.2. Let $\mathbb{R}$ be the set of all real numbers. Define $x * y:=x-y+\sqrt{2}$. Then $(\mathbb{R} ; *, \sqrt{2})$ is a quadratic $Q$-algebra.

EXAMPLE 4.3. Let $\mathscr{K}:=\mathrm{GF}\left(p^{n}\right)$ be a Galois field. Define $x * y:=x-y+e, e \in \mathscr{K}$. Then $(\mathscr{K} ; *, e)$ is a quadratic $Q$-algebra.

THEOREM 4.4. Let $X$ be a field with $|X| \geq 3$. Then every quadratic $Q$-algebra on $X$ is a (quadratic) $Q S$-algebra.

Proof. Let $(X ; *, e)$ be a quadratic $Q$-algebra. Then $x * y=x-y+e$ for any $x, y \in X$, and hence

$$
\begin{aligned}
(x * y) *(x * z) & =(x-y+e) *(x-z+e) \\
& =(x-y+e)-(x-z+e)+e \\
& =z-y+e=z * y,
\end{aligned}
$$

completing the proof.

REMARK 4.5. Usually a nonquadratic $Q$-algebra need not be a $Q S$-algebra. See the following example. 
EXAMPLE 4.6. Consider the $Q$-algebra $(X ; *, 0)$ in Example 2.2. This algebra is not a $Q S$-algebra, since $(3 * 1) *(3 * 2)=3 \neq 0=2 * 1$.

COROLLARY 4.7. Let $X$ be a field with $|X| \geq 3$. Then every quadratic $Q$-algebra on $X$ is a BCI-algebra.

Proof. It is an immediate consequences of Theorems 2.5 and 4.4 .

THEOREM 4.8. Let $X$ be a field with $|X| \geq 3$. Then every quadratic $Q$-algebra $(X ; *, e)$ is $p$-semisimple. Furthermore, if $\operatorname{char}(X) \neq 2$, then $G(X)=B(X)$.

Proof. Notice that $B(X)=\{x \in X \mid e * x=e\}=\{x \in X \mid e-x+e=e\}=\{x \in$ $X \mid e-x=0\}=\{e\}$, that is, $(X ; *, e)$ is $p$-semisimple. Also, if $\operatorname{char}(X) \neq 2$, then 2 is invertible in $X$ and $G(X)=\{x \in X \mid e * x=x\}=\{x \in X \mid e-x+e=x\}=\{x \in X \mid$ $2 e=2 x\}=\{x \in X \mid e=x\}=\{e\}$. Of course, if $\operatorname{char}(X)=2$, then $2 e=2 x=0$ for all $x \in X$, whence $G(X)=X$.

This shows that there is a large class of examples of $p$-semisimple $Q S$-algebras obtained as quadratic $Q$-algebras.

THEOREM 4.9. Let $X$ be a field with $|X| \geq 3$. Then every quadratic $Q$-algebra on $X$ is isomorphic to every other such algebra defined on $X$.

Proof. Let $x * y:=x-y+e_{1}$ and $x *^{\prime} y:=x-y+e_{2}$, where $e_{1}, e_{2} \in X$. Let $\pi(x):=x+\left(e_{2}-e_{1}\right)$, for all $x \in X$. Then $\pi(x * y)=\left[(x-y)+e_{1}\right]+\left(e_{2}-e_{1}\right)=$ $(x-y)+e_{2}=\left(x+\left(e_{2}-e_{1}\right)\right)+\left(y+\left(e_{2}-e_{1}\right)\right)+e_{2}=\pi(x) *^{\prime} \pi(y)$, whence the fact that $\pi^{-1}(x)=x+\left(e_{1}-e_{2}\right)$ yields the conclusion that $\pi$ is an isomorphism of $Q$-algebras.

THEOREM 4.10. Let $X$ be a field with $|X| \geq 3$. Then every quadratic $Q$-algebra $(X ; *, e)$ determines the abelian group $(X,+)$ via the definition $x+y=x *(e-y)$.

Proof. Note that $x *(e-y)=x-(e-y)+e=x+y$ returns the additive operation of the field $X$, which is an abelian group.

Not every quadratic $Q$-algebra $(X ; *, e), e \in X$, on a field $X$ with $|X| \geq 3$ need be a $B C K$-algebra, since $((x * y) *(x * z)) *(z * y)=e+(y-z) \neq e$ in general.

Problem 4.11. Construct a cubic $Q$-algebra which is not quadratic. Verify that among such cubic $Q$-algebras there are examples which are not $Q S$-algebras. Furthermore, the question whether there are non- $p$-semisimple cubic $Q$-algebras is also of interest.

\section{REFERENCES}

[1] S. S. Ahn and H. S. Kim, On QS-algebras, J. Chungcheong Math. Soc. 12 (1999), 33-41.

[2] Q. P. Hu and X. Li, On BCH-algebras, Math. Sem. Notes Kobe Univ. 11 (1983), no. 2, part 2, 313-320. MR 86a:06016. Zbl 579.03047.

[3] _ On proper BCH-algebras, Math. Japon. 30 (1985), no. 4, 659-661. MR 87d:06042. Zbl 583.03050.

[4] K. Iséki, On BCI-algebras, Math. Sem. Notes Kobe Univ. 8 (1980), no. 1, 125-130. MR 81k:06018a. Zbl 0434.03049. 
[5] K. Iséki and S. Tanaka, An introduction to the theory of BCK-algebras, Math. Japon. 23 (1978), no. 1, 1-26. MR 80a:03081. Zbl 385.03051.

[6] Y. B. Jun, E. H. Roh, and H. S. Kim, On BH-algebras, Sci. Math. 1 (1998), no. 3, 347-354. MR 2000d:06026. Zbl 928.06013.

[7] J. Neggers and H. S. Kim, On B-algebras, in preparation.

[8] _ On d-algebras, Math. Slovaca 49 (1999), no. 1, 19-26. CMP 1804469. Zbl 943.06012.

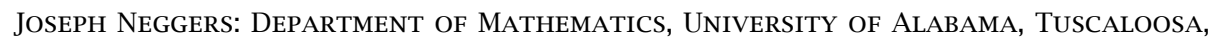
AL 35487-0350, USA

E-mail address: jneggers@gp.as.ua.edu

Sun Shin Ahn: Department of Mathematics Education, Dongguk University, SeOUl 100-715, KOREA

E-mail address: sunshine@dgu.ac. kr

HeE Sik Kim: Department of Mathematics, Hanyang National University, SeOUl 133-791, KOREA

E-mail address: heekim@hanyang.ac.kr 


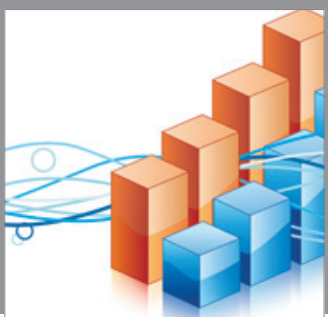

Advances in

Operations Research

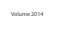

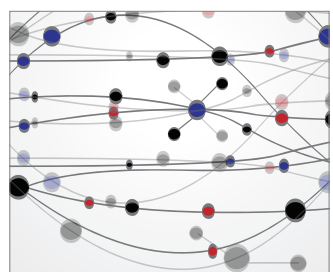

\section{The Scientific} World Journal
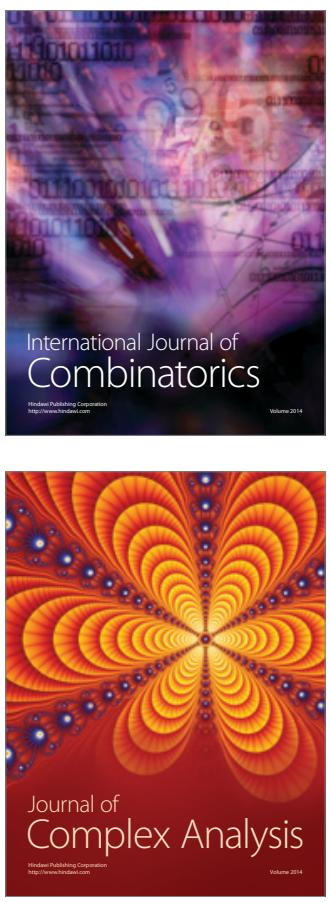

International Journal of

Mathematics and

Mathematical

Sciences
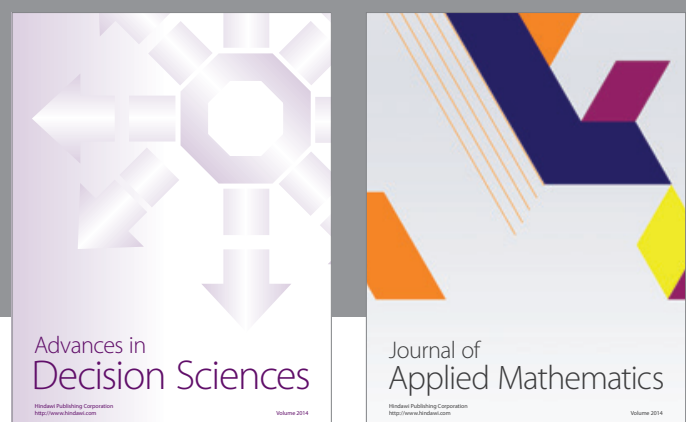

Journal of

Applied Mathematics
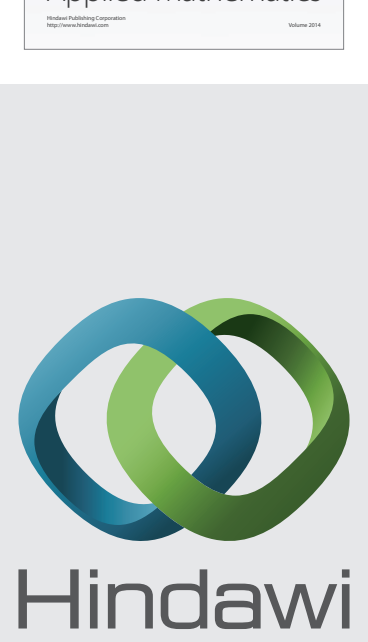

Submit your manuscripts at http://www.hindawi.com
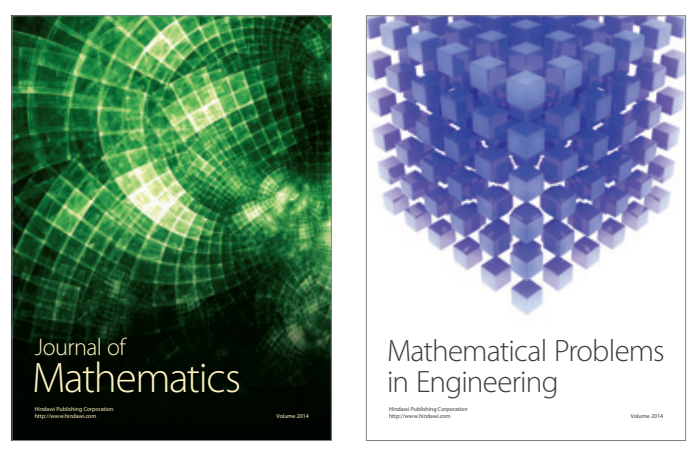

Mathematical Problems in Engineering
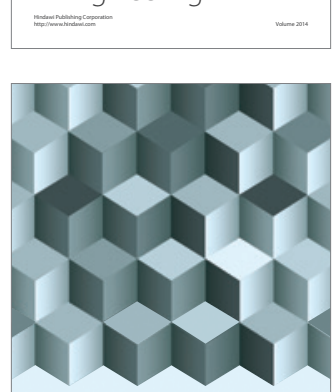

Journal of

Function Spaces
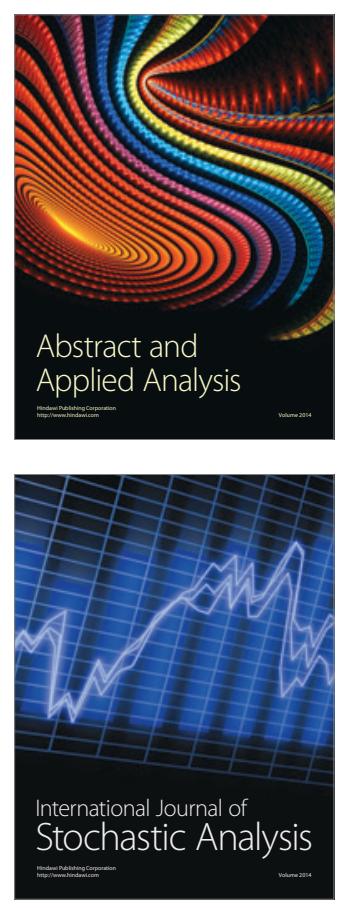

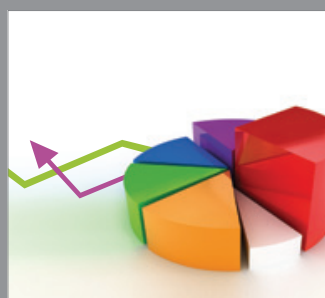

ournal of

Probability and Statistics

Promensencen
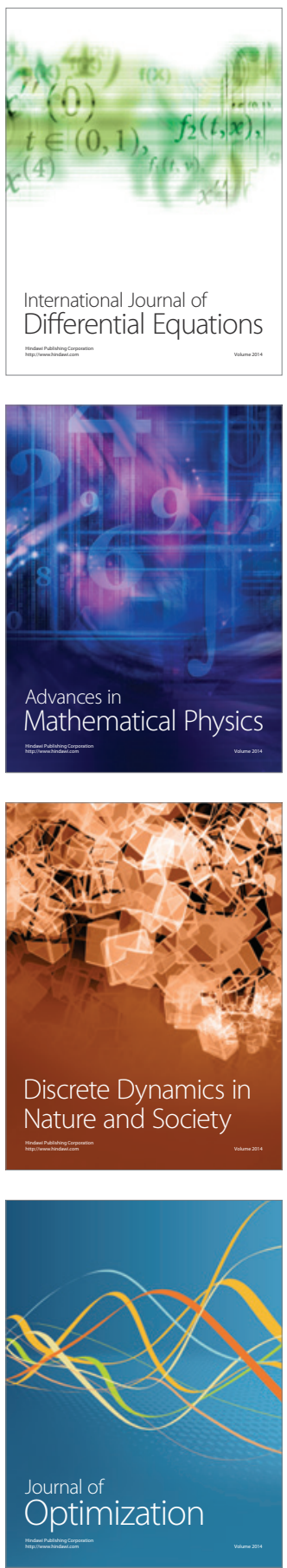